\title{
What was the ancestral function of decidual stromal cells? A model for the evolution of eutherian pregnancy
}

Arun Rajendra Chavan¹,2, Bhart-Anjan S. Bhullar³, and Günter P. Wagner 1, 2, 4, 5

1. Department of Ecology and Evolutionary Biology, Yale University, New Haven, Connecticut 06511, USA

2. Yale Systems Biology Institute, Yale University, West Haven, Connecticut 06516, USA

3. Department of Geology and Geophysics, Yale University, New Haven, Connecticut 06511, USA

4. Department of Obstetrics, Gynecology and Reproductive Sciences, Yale Medical School

5. Department of Obstetrics and Gynecology, Wayne State University, Detroit MI. 


\begin{abstract}
In human and mouse, decidual stromal cells (DSC) are necessary for the establishment (implantation) and the maintenance of pregnancy by preventing inflammation and the immune rejection of the semi-allograft conceptus. DSC originated along the stem lineage of eutherian mammals, coincidental with the origin of invasive placentation. Surprisingly, in many eutherian lineages decidual cells are lost after the implantation phase of pregnancy, making it unlikely that DSC are necessary for the maintenance of pregnancy in these animals. In order to understand this variation, we review the literature on the fetal-maternal interface in all major eutherian clades Euarchontoglires, Laurasiatheria, Xenarthra and Afrotheria, as well as the literature about the ancestral eutherian species. We conclude that maintaining pregnancy may not be a shared derived function of DSC among all eutherian mammals. Rather, we propose that DSC originated to manage the inflammatory reaction associated with invasive implantation. We envision that this happened in a stem eutherian that had invasive placenta but still a short gestation. We further propose that extended gestation evolved independently in the major eutherian clades explaining why the major lineages of eutherian mammals differ with respect to the mechanisms maintaining pregnancy.
\end{abstract}

Keywords: Decidual stromal cells, Endometrial stromal cells, Eutheria, Extended gestation, origin of placental mammals 


\section{What was the ancestral function of decidual stromal cells?}

A model for the evolution of eutherian pregnancy

Arun Rajendra Chavan¹, 2, Bhart-Anjan S. Bhullar³, and Günter P. Wagner ${ }^{1,2,4,5}$

1. Department of Ecology and Evolutionary Biology, Yale University, New Haven, Connecticut 06511, USA

2. Yale Systems Biology Institute, Yale University, West Haven, Connecticut 06516, USA

3. Department of Geology and Geophysics, Yale University, New Haven, Connecticut 06511, USA

4. Department of Obstetrics, Gynecology and Reproductive Sciences, Yale Medical School

5. Department of Obstetrics and Gynecology, Wayne State University, Detroit MI. 


\begin{abstract}
In human and mouse, decidual stromal cells (DSC) are necessary for the establishment (implantation) and the maintenance of pregnancy by preventing inflammation and the immune rejection of the semi-allograft conceptus. DSC originated along the stem lineage of eutherian mammals, coincidental with the origin of invasive placentation. Surprisingly, in many eutherian lineages decidual cells are lost after the implantation phase of pregnancy, making it unlikely that DSC are necessary for the maintenance of pregnancy in these animals. In order to understand this variation, we review the literature on the fetal-maternal interface in all major eutherian clades Euarchontoglires, Laurasiatheria, Xenarthra and Afrotheria, as well as the literature about the ancestral eutherian species. We conclude that maintaining pregnancy may not be a shared derived function of DSC among all eutherian mammals. Rather, we propose that DSC originated to manage the inflammatory reaction associated with invasive implantation. We envision that this happened in a stem eutherian that had invasive placenta but still a short gestation. We further propose that extended gestation evolved independently in the major eutherian clades explaining why the major lineages of eutherian mammals differ with respect to the mechanisms maintaining pregnancy.
\end{abstract}

Keywords: Decidual stromal cells, Endometrial stromal cells, Eutheria, Extended gestation, origin of placental mammals 


\section{Introduction}

Evolution of pregnancy is one of the salient attributes of eutherian mammals. Distinctive features of eutherian pregnancy include invasive placentation (at least ancestrally), extended gestation, maternal recognition of pregnancy, and the origin of decidual stromal cells (DSC).

DSCs are a novel cell-type that originated along the stem lineage of eutherian mammals (Mess and Carter 2006, Kin, Maziarz et al. 2014). In many eutherian mammals, DSC differentiate from a population of fibroblast-like cells in the uterus called Endometrial Stromal Fibroblasts (ESF) in a process called decidualization. In many eutherian mammals ESF decidualize during pregnancy, and in some eutherians, including humans, also during the secretory phase of a sterile sexual cycle (Gellersen and Brosens 2014). Human ESF decidualize in response to progesterone and cyclic-AMP (cAMP).

Differentiation of DSC from ESF involves extensive reprogramming of gene regulatory status (Brar, Handwerger et al. 2001) and changes to the genome-wide patterns of histone-modification (Tamura, Ohkawa et al. 2014). They also acquire a distinct cellular morphology - increased size, globular or polygonal appearance compared to the spindle-like appearance of their precursor fibroblasts, and increased accumulation of fat and glycogen granules as well as secretion of extracellular matrix (Mossman 1937).

Functions of decidual stromal cells

The functions of DSC have been studied extensively in model systems such as rodents and in-vitro grown human endometrial stromal cells. In human and mouse DSC form a physical barrier between the invading syncytio-trophoblast and the maternal tissue. Failure to form this barrier in human leads to a pathological invasion of myometrium by the trophoblast, a condition called placenta accreta that can be fatal to the mother (Gersell and Kraus 2011). The presence of glycogen and lipid granules suggests a nutritive function toward the fetus (Mossman 1937). In addition DSC produce a variety of signaling molecules including prolactin, prostaglandins, relaxin, IGFBP1 (Insulin-Like Growth Factor Binding Protein 1) and many more. These hormones and paracrine factors are important for maintaining maternal physiology in a state conducive to pregnancy (Wooding and Burton 2008). In humans there is also evidence that the decidua plays a role in embryo selection, ensuring that only viable blastocysts can implant successfully (Macklon and Brosens 2014).

Eutherian blastocyst implantation is an inflammatory process (Wang and Dey 2006, Dekel, Gnainsky et al. 2014) and inflammation is necessary for successful implantation. Never the less soon after implantation the local endometrial environment becomes anti-inflammatory, a step necessary for the maintenance of 
pregnancy, mediated, in part, by a switch of the decidual cell cytokine profile (Salker, Nautiyal et al. 2012).

The fetus expresses paternal antigens that can be identified by maternal immune system as 'non-self'. Yet, the semi-allograft fetus is not rejected by the maternal immune system. How this fetal-maternal immune tolerance is brought about is a long-standing puzzle in reproductive biology. In the last few years, DSC have been recognized as critical mediators of immunological tolerance at the fetal-maternal interface in human and mouse (Erlebacher 2013).

In the mouse, through epigenetic silencing of the chemokines, $\mathrm{Cxcl9}$ (C-X-C Motif Chemokine 9) and Cxcl10 (C-X-C Motif Chemokine 10), DSC limit the influx of cytotoxic T-cells into the endometrium, thus minimizing the interactions between the trophoblast and effectors of the immune system (Nancy, Tagliani et al. 2012). Uterine variants of natural killer cells (uterine Natural Killer cells or uNK cells) and macrophages are distinct from their circulating counterparts in being less cytotoxic and active players in remodeling of uterine vasculature to accommodate invasive placentation. Acquisition of their distinct status in the endometrium is mediated by Interleukin-15 (IL15) secreted by decidual cells (Kitaya, Yasuda et al. 2000, Erlebacher 2013). Conditioned medium from DSC, supplemented with IL15, converts peripheral natural killer cells to their uterine phenotype (Keskin, Allan et al. 2007), and co-culture of CD34-positive hematopoietic precursor cells with DSC converts the precursor cells into uNK cells (Vacca, Vitale et al. 2011). Decidualization in mouse creates an environment that prevents the growth of lymphatic vasculature in the endometrium, trapping dendritic cells in the endometrium (Collins, Tay et al. 2009). Dendritic cells are antigen-presenting cells that must traverse to a lymph node through lymphatic vasculature to present antigens to lymphocytes. This important event in the activation of the adaptive immune system is thus interrupted by decidualization, at least in mouse.

Evidently, in human and mouse, DSC play a critical role in modulation of the uterine environment to facilitate and maintain the extended gestation in the face of immunological and physiological challenges of an invasive placenta.

\section{Evolutionary origin of decidual stromal cells}

DSC originated along the eutherian stem lineage as inferred by phylogenetic ancestral state reconstruction (Mess and Carter 2006). The evolution of functional interactions between certain transcription factors necessary for DSC differentiation also occurred at the same time in phylogeny, supporting this inference. DSC differentiation is dependent on functional cooperative interactions between HOXA11 (Homeobox A11) and F0X01 (Forkhead Box 01) (Lynch, Brayer et al. 2009), and between FOX01 and CEBPB (CCAAT/Enhancer Binding Protein, Beta) (Christian, Zhang et al. 2002). These cooperative interactions evolved along the stem lineage of eutherian mammals: reconstructed ancestral eutherian versions of these transcription factors have the ability to up-regulate the expression of DSC 
markers, while the reconstructed ancestral therian versions lack this ability as do the proteins of outgroup species, opossum, platypus and chicken (HOXA11: Brayer, Lynch et al. 2011, CEBPB: Lynch, May et al. 2011).

While mammalian viviparity and direct fetal-maternal contact and interaction likely evolved in the stem lineage of therian mammals (i.e. prior to the ancestor of both marsupial and eutherian mammals), the fetal-maternal interaction is qualitatively different between metatherian (marsupial) and eutherian mammals. Highly invasive forms of placentation that lead to a sustainable accommodation of the fetal allograft are unique to eutherian mammals (Mossman 1987).

Placentation has been categorized into three major types based on the maternal tissue coming in direct contact with the trophoblast: epitheliochorial, endotheliochorial and haemochorial. Epitheliochorial (trophoblast is in contact with the luminal epithelium of endometrium) placentation is non-invasive because the fetal tissue does not breach the uterine luminal epithelium (Grosser 1927). It is found in cattle, sheep, pig and horse and their relatives like dolphins and whales. Endotheliochorial (trophoblast is in contact with maternal endothelium) and haemochorial (trophoblast is in contact with maternal blood) are invasive forms because fetal tissue breaches the luminal epithelium and establishes a direct contact with endometrial stroma. Endotheliochorial placentation is found in carnivores, elephant etc. and haemochorial placentation is found in primates, rodents, armadillo etc. (Mossman 1987). Phylogenetic ancestral state reconstructions have inferred that the eutherian ancestor possessed an invasive form of placentation. Disagreement remains whether it was endotheliochorial (Mess and Carter 2006) or haemochorial (Wildman, Chen et al. 2006, Elliot and Crespi 2009); what is clear, however, is that placentation was invasive in the eutherian ancestor (Martin 2008). In either case, the ancestor of eutherians had a placenta where the trophoblast of the conceptus was in direct contact with the endometrial stroma. See Figure 1 for a phylogeny of mammals and Figure 2 for the evolution of mode of placentation in Eutheria.

DSC are typically found in species that exhibit invasive placentation, with the possible exception of armadillo and other xenarthrans, discussed below. After their origin in the eutherian stem lineage, DSC are reconstructed to have been lost in the lineage leading from the laurasiatherian ancestor, the same lineage in which invasive mode of placentation was lost (Mess and Carter 2006).

When DSC originated is relatively clear, based on multiple lines of evidence mentioned above. However, which evolutionary forces drove their origin, what their ancestral function was, and which ontogenetic and gene regulatory changes made their origin possible are open questions. It is important to address these questions for at least two reasons. First, how and why evolutionary novelties such as novel cell-types originate is a fundamental question in evolutionary biology (Arendt 2008, Wagner and Lynch 2010). Secondly, understanding how and why DSC originated can inform efforts to dissect the mechanistic basis of reproductive pathologies. 
In order to understand the origin and ancestral function of DSC, research on rodent and primate model systems needs to be supplemented with data on the other major lineages of eutherians, in particular Afrotheria (tenrec, hyrax, elephant, manatee etc.) and Xenarthra (armadillo, anteater, sloth etc.). Primates and rodents are members of one of the four major eutherian clades, Euarchontoglires. Given that DSC originated in the eutherian stem lineage, it is imperative that any inferences concerning their origin be drawn from studies on taxa that bracket the entire diversity of eutherian descent: Xenarthra, Afrotheria and Laurasiatheria, in addition to Euarchontoglires.

To this end, we reviewed the literature on DSC in Eutheria, with specific attention to Xenarthra (armadillo) and Afrotheria (tenrec and hyrax). A surprising observation about DSC is that they are generally present in the peri-implantation phase, but tend to disappear in later stages of pregnancy. The latter observation is incompatible with the hypothesis that maintenance of extended pregnancy is a shared derived eutherian function of DSC.

\section{The life cycle of DSC and its implications for their ancestral function}

\section{DSC do not generally persist until the end of gestation}

In species that have decidual cells, their numbers dwindle as pregnancy progresses. Mossman has pointed this out earlier in case of human and rodents (Mossman 1937). Here we call attention to this phenomenon in a select set of eutherian species representing Euarchontoglires, Xenarthra, Afrotheria and Laurasiatheria.

\section{a. Euarchontoglires (Rat, Rattus norvegicus)}

In rat, similar to the situation in mouse, there are two sites of decidualization in the uterus. Primary decidualization takes place on the antimesometrial side in response to the implantation of the blastocyst. It is followed by secondary decidualization on the mesometrial side (Abrahamsohn and Zorn 1993). Mesometrial decidua forms the decidua basalis. Antimesometrial decidua encapsulates the conceptus, thus forming the decidua capsularis. The thickness of antimesometrial decidua consistently decreases from day 8 of gestation before it completely disappears by day 18, 2 to 3 days prior to parturition (Dickson 1979). Similarly mesometrial decidua begins regression on day 14 and continues to regress until the end of gestation (Dai, Moulton et al. 2000, Furukawa, Hayashi et al. 2011). Several studies have shown that this regression is mediated by apoptotic cell death (Gu, Jow et al. 1994, Dai, Moulton et al. 2000, Shooner, Caron et al. 2005) (see Figure 3a).

b. Xenarthra (Nine-banded armadillo, Dasypus novemcinctus) 
In the nine-banded armadillo (Dasypus novemcinctus), the trophoblast comes in contact with the endometrial stromal cells at the time of implantation. The question whether armadillo has DSC is still not completely settled. Enders and colleagues (Enders, Buchanan et al. 1958) described a small number of cells that have the histological signs of DSC, but there certainly is no compact layer of DSC comparable to the situation in rodents and primates, and other authors deny the existence of a decidua (Benirschke 2011). In the definitive (fully developed) placenta, however, the trophoblast is in close contact with the myometrium; i.e. there is no decidua basalis (Enders, Buchanan et al. 1958). Our immunohistochemical investigation of armadillo placenta corroborates this observation (Chavan et al. in prep.). The only remnants of the endometrial stroma are thin bands separating the lobules of the placenta and a thin band encapsulating the placenta. Enders and colleagues (Enders, Buchanan et al. 1958) have called the latter 'the endometrial arcade'; it is not decidua capsularis because it does not surround the fetus, but only the placenta. This situation develops because the trophoblast does not invade the endometrium on a broad front, like in mouse or human, but sends finger like projections into pre-formed blood sinuses, which then branch to form a villous tree inside the maternal blood space (Enders and Carter 2012). In essence, there is substantial amount of endometrial stroma present at the time of implantation but all of it disappears from the basal side of the definitive placenta, leaving the myometrium in close proximity to the trophoblast (see Figures $3 \mathrm{~b} \& 3 \mathrm{c}$ ). Whether the endometrial arcade contains DSC is still unclear. A similar situation has been described for another xenarthran species, the ant eater Tamandua tetradactylus (Becher 1931). Early in pregnancy a broad decidualization of the stroma is found but at the site of placentation the decidua are completely lost in later stages of gestation.

c. Afrotheria (Rock hyrax, Procavia capensis \& Lesser hedgehog tenrec, Echinops telfairi)

According to Thursby-Pelham's description of the uterus of hyrax, Procavia capensis, the decidual layer is at its thickest in the earliest specimen studied (specimen 3, uterine diameter $8 \mathrm{~mm}$ ), which gradually thins out in specimens at later stages (specimen 4 , uterine diameter $15 \mathrm{~mm}$ and specimen 5 , uterine diameter 17mm) (Thursby-Pelham 1925). Our examination of histological preparations of hyrax (P. capensis) uterus (ID 20487 from Mossman Developmental Collection, Zoological Museum, University of Wisconsin, Madison) confirms these results (see Figures $3 \mathrm{~d} \& 3 \mathrm{e}$ ).

Carter and colleagues have described the definitive placenta of tenrec, Echinops telfairi, and its development (Carter, Blankenship et al. 2004, Carter, Blankenship et al. 2005). During the development of the placenta, stromal cells can be seen in the endometrium, which are potentially DSC. Carter and colleagues do not call these cells 'decidual' based on their histological appearance, but further investigation is needed to resolve their identity. The 
possibility is open that these cells are differentiated decidual stromal cells (by their gene regulatory identity) but histologically inconspicuous. Despite their equivocal identity as ESF or DSC, endometrial stromal cells are lost entirely in the later stages of pregnancy, eliminating the possibility of persistence of any DSC through gestation (see Figures 3f \& 3g).

d. Laurasiatheria (European mole, Talpa europaea; Indian false vampire bat, Megaderma lyra lyra; and Neotropical disc-winged bat, Thyroptera tricolor spix):

Laurasiatherian clades, Carnivora (e.g. cat, mink), Chiroptera (bats), and Eulipotyphla (e.g. European mole), have members with invasive placentation. Carnivores are termed as 'deciduate' species because maternal tissue, mostly glandular epithelium and endothelium, is shed at birth. However, their ESF do not seem to undergo decidualization based on histological criteria (Mossman 1987). The literature on DSC of Eulipotyphla and Chiroptera suggests that DSC are present around the peri-implantation period, but undergo degeneration in later stages; sometimes all of them, e.g. in case of the Indian false vampire bat, Megaderma lyra lyra, bringing the placental villi and myometrium in apposition (Indian false vampire bat: Gopalakrishna and Khaparde 1978, Neotropical discwinged bat: Wimsatt and Enders 1980, European mole: Malassine and Leiser 1984).

It is clear that in these species, drawn from all major clades of Eutheria, DSC are present, with possible exceptions, and the layer of decidual cells is at its thickest at the time of implantation. DSC tend to disappear in the later stages of pregnancy. This is particularly striking in armadillo and tenrec, in which decidua basalis seems to be completely abolished once the definitive placenta is established - a situation that can be called a 'physiological placenta accreta' - a condition clearly pathological in human but normal in these animals.

\section{The ancestral function of DSC is its role during implantation}

The taxonomic distribution of the life cycle of DSC described above suggests that the presence of DSC is a shared derived state of eutherian mammals. Furthermore the evidence suggests that later stages of pregnancy are not homologous (Swaggart, Pavlicev et al. 2015). This leads to the conclusion that, ancestrally, DSC had a role to play during early stages of pregnancy. Their endocrine and immune functions in later stages of pregnancy seem to be limited to Euarchontoglires rather than being general to Eutheria. When this observation is placed in the context of what we know about mammalian evolution, the following model emerges. Our model is consistent with a similar argument proposed by Swaggart and colleagues, namely that the later stages in the pregnancy of eutherian taxa are not homologous (Swaggart, Pavlicev et al. 2015). 
We propose that, in the eutherian stem lineage, gestation was shorter than or at most as long as the sterile sexual cycle, i.e. the ancestral eutherian did not have 'extended gestation' similar to the situation in basal marsupial lineages, e.g. the opossums (Renfree 1994).

To our knowledge, a term for length of gestation in relation to the length of the sterile sexual cycle doesn't exist. Gestation longer than sterile sexual cycle is often referred to as 'extended gestation', but this term can also be used to describe the absolute length of gestation, not relative to that of the sterile sexual cycle. We would like to propose the terms 'intra-cyclic gestation' and 'trans-cyclic gestation' to refer to gestation shorter and longer than the sterile sexual cycle, respectively. We follow this terminology throughout this article from here on. See Figure 4 for an illustration explaining intra-cyclic and trans-cyclic gestations.

We propose that trans-cyclic gestation originated independently in the four major eutherian lineages. This proposal can explain the wide range of differences in the role of DSC reviewed above as well as other differences briefly summarized below. It is based on the fact that species in basal marsupial lineages have short intra-cyclic gestation, that the members of the eutherian stem lineage and probably also the eutherian ancestor were small animals, and that the four major lineages of eutherian arose in a very short period of intense radiation (evidence reviewed below).

This scenario implies that when DSC originated in the stem eutherian lineage, gestation likely involved only a short post-implantation phase. The ancestral function of DSC was, therefore, limited to the implantation process, perhaps to dampen the inflammatory response elicited by invasive implantation (Mor, Cardenas et al. 2011). If eutherian pregnancy was extended beyond the length of sterile sexual cycle independently in major eutherian lineages, the endocrine and immune mechanisms for maintaining trans-cyclic gestation were also acquired independently in each of the major lineages and thus differ in the way pregnancy is maintained. From this model we infer that the role of DSC in maintaining pregnancy in primates and rodents evolved in the stem lineage of the Euarchontoglires rather than in the stem lineage of Eutheria.

\section{The paleontological and comparative physiological evidence}

The validity of the model explained above hinges upon the assumption that the eutherian ancestor had an intra-cyclic gestation. In this section we present evidence that substantiates this assumption.

Trans-cyclic gestation probably originated independently in major eutherian lineages 
Marsupial pregnancy is intra-cyclic (McAllan 2011). Maternal hormonal cycle is not altered by the presence of a fetus; there is no so-called 'maternal recognition of pregnancy' except in highly derived macropodids, e.g. wallabies (Renfree 1994). The condition in macropodids is not a shared derived character of marsupials (Freyer, Zeller et al. 2003).

The situation in marsupials is in sharp contrast to that in eutherians. Gestation going beyond the length of sterile sexual cycle, trans-cyclic gestation, is considered to be a hallmark of eutherian pregnancy. Eutherian gestation is always trans-cyclic. There is maternal recognition of pregnancy: maternal physiology is altered by the presence of the fetus; in particular, progesterone production is sustained through gestation (Bazer and Spencer 2011).

The evidence detailed below supports our argument that trans-cyclic gestation had multiple independent origins.

\section{a. The eutherian ancestor had a short gestation}

The fossil record suggests that the eutherian ancestor was a very small animal, and that body size increased independently in several lineages ( 0 'Leary, Bloch et al. 2013). The high end of reconstructed ancestral sizes (in the 100-200 g range) is likely biased by the secondary body size enlargements strongly suggested to have taken place along the monotreme and marsupial stems. In fact, fossil stem monotremes and stem metatherians are very small, as are stem eutherians (KielanJaworowska, Cifelli et al. 2004). These would be closer to the 1-15 g range of small extant insectivores. We think that this is a conservative estimate since remains of larger animals are more likely to be recovered than those of very small animals.

Moreover, there is direct evidence that body sizes increased along each of the major placental lineages - not just on the stems of the four "main" clades but even on the various stem lineages of smaller "order level" clades within these. This pattern of body size increase was noticed long ago and given the term "Cope's Rule" (Hone and Benton 2005); however, the concept is vague and not well controlled phylogenetically. The pattern of evolution along the stems of major placental lineages is further obfuscated by the fact that very few of the thousands of known Paleogene mammal taxa have been rigorously placed phylogenetically. Much additional work remains to be done. Nevertheless, along the earliest parts of the stem lineages of the various placental clades are small-bodied animals. The afrotherian and xenarthran records are unfortunately poor in the early Paleogene. Among Euarchontoglires, rodents have retained small body size for the most part, as have tree shrews and dermopterans, successive sisters to the larger-bodied Primates. For the most part, Eulipotyphla and Chiroptera, "insectivorans" and bats, remain small-bodied and their fossil stem taxa are small-bodied as well (Simmons, Seymour et al. 2008). Early possible stem perissodactyls ("condylarths"), early stem pangolins, i.e. scaly anteaters ("palaeanodonts"), and early stem carnivorans (for instance from the Eocene of Germany) are shrew or mouse size (Rose and Archibald 
2005). The stem artiodactyl record is not as complete, but the earliest stem artiodactyls, while not as tiny as the stem members of other lineages, are roughly the size of large rabbits (Rose 1982, Cooper, Thewissen et al. 2012). A general pattern thus emerges of repeated instances of extreme body enlargement.

Life history studies have consistently found a positive relationship between body weight (which scales with body size) and the length of gestation (Kihlstrom 1972, Martin and Maclarnon 1985). With the use of phylogenetic comparative methods controlling for the effects of shared history, this relationship is less steep than was previously believed. The allometric scaling exponent is 0.18 to 0.2 by ordinary least squares method but 0.07 to 0.1 by phylogenetic generalized least squares method (Clauss, Dittmann et al. 2014). However, there is little reason to doubt the general relationship between body size and gestation length outside of lower taxonomic levels, given that most time during long gestations is dedicated to fetal growth, and thus has to relate to neonatal size.

Given the small body sizes of the eutherian ancestor and the ancestors of the major eutherian clades, and the relationship between body size and gestation length, we infer that these animals likely had short gestations. Whether this amounts to intracyclic gestation is not directly testable, but at least large body size in the eutherian ancestor would be incompatible with the hypothesis of intra-cyclic gestation.

The idea that the eutherian ancestor gave birth to altricial neonates (typically defined by lack of hair and closed eyes at birth and the need for extensive parental care) has existed for a long time (Portmann 1938). This idea has been consistently supported by several studies using phylogenetic ancestral state reconstruction (Mess and Carter 2006, O'Leary, Bloch et al. 2013). Polarization of the character is provided in part by the highly altricial nature of monotreme hatchlings, which are similar in many ways to marsupial neonates. The fact that altricial neonates are born after a relatively short gestation, and depend largely on lactation for their growth, is also suggestive of a short gestation in the eutherian ancestor.

Admittedly, this reasoning alone doesn't lead us to the inference that the eutherian ancestor had an intra-cyclic gestation (i.e. gestation shorter than the sterile sexual cycle). However, we must reflect upon the inferred short gestation of the eutherian ancestor in the context of the ancestral therian mode of reproduction, which most likely did not involve trans-cyclic gestation. More likely than the origin of transcyclic gestation in the eutherian stem lineage is the possibility that characters such as invasive placentation and DSC originated first, paving the way for the evolution of much longer trans-cyclic gestation. Once the problem of inflammation caused by implantation was solved, in part, through the anti-inflammatory role of DSC and progesterone, further extension of gestation was possible leading to trans-cyclic gestation.

b. Mechanisms involved in maintenance of trans-cyclic gestation are highly variable 
Corpus luteum, the remnant of an ovarian follicle upon ovulation, secretes progesterone. In eutherian mammals, a successful pregnancy requires sustained progesterone production, well beyond the life span of the corpus luteum of a sterile sexual cycle. This can be achieved in two ways: by extension of the life span of corpus luteum or by another organ taking over the responsibility of progesterone secretion after the corpus luteum has regressed. All eutherian taxa use either one of these two mechanisms or both to maintain their pregnancy beyond the length of a sterile sexual cycle. However, the means by which they activate these mechanisms are remarkably different (Bazer and Spencer 2011). See Figure 5 for a summary of this section.

Corpus luteum can be rescued from regression either by promotion of its growth through luteotropic signals or by inhibition of luteolysis through anti-luteolytic signals. In a sterile sexual cycle, prostaglandins, most often from the uterus, lead to luteolysis. Anti-luteolytic signals act to negate the effects of prostaglandins.

In Euarchontoglires, typically the life span of corpus luteum is extended by luteotropic signals, which may or may not be followed by a luteo-placental shift in progesterone production. The luteotropic signal in human, human Chorionic Gonadotrophin (hCG) from the trophoblast, promotes growth of corpus luteum through part of gestation, after which the placenta takes over progesterone production. The luteotropic signal in rodents is prolactin, likely secreted by the decidua or the pituitary. It promotes growth of corpus luteum through the entire length of gestation, without a luteo-placental shift. Rabbits also do not have a luteoplacental shift. They make use of an unidentified luteotropic signal of placental origin in addition to estradiol (E2) as the anti-luteolytic signal (Bazer and Spencer 2011).

In Laurasiatheria, the mechanisms are more variable than in Euarchontoglires. Artiodactyls typically use anti-luteolytic signals, along with or without a shift in the organ of progesterone production. In pecoran ruminants (sheep, cattle, goat) IFN- $\tau$ secreted by the conceptus extends the life span of corpus luteum by suppressing endometrial prostaglandin-mediated luteolysis. Pigs also use an anti-luteolytic signal but it is E2 secreted by the pig trophoblast, which converts the uterine prostaglandin secretion from endocrine to exocrine. This prevents the entry of prostaglandin into the bloodstream, and therefore it cannot reach the corpus luteum (Bazer and Spencer 2011).

Horse (a perissodactyl) uses equine Chorionic Gonadotropin (eCG) as a luteotropic signal, which probably induces production of accessory corpora lutea. Accessory corpora lutea are ovarian follicles that luteinize without ovulation and contribute to progesterone production. The placenta primarily carries out progesterone production in late gestation after regression of primary and accessory corpora lutea (Allen 1984). 
Dog (a carnivore) possibly does not have a signal from the conceptus for extension of the life span of the corpus luteum, given that the life span of corpus luteum is not altered during pregnancy in comparison to pseudo-pregnancy (Bazer and Spencer 2011).

In armadillo (a xenarthran), the corpus luteum regresses during blastocyst dormancy. After implantation progesterone production is taken over by the adrenals of the fetus (Nakakura, Czekala et al. 1982), possibly supplemented by the placenta (Labhsetwar and Enders 1968).

Elephant (an afrotherian) shows signs of neither a luteo-placental shift nor a luteotropic or anti-luteolytic signal. It develops accessory corpora lutea that secrete progesterone through the length of gestation (Lueders, Niemuller et al. 2012, Stansfield and Allen 2012).

In sum, sustained production of progesterone, past the life span of corpus luteum of a sterile sexual cycle, is the pivotal modification of maternal physiology during pregnancy that makes trans-cyclic gestation possible. Highly divergent mechanisms involved in bringing about this outcome strongly suggest the possibility that transcyclic gestation is not an ancestral character of Eutheria, but that it originated independently in various lineages, as also proposed by Swaggart and colleagues (Swaggart, Pavlicev et al. 2015).

\section{c. Epipubic bones in stem Eutheria}

Epipubic bones are a pair of bones projecting anterio-ventrally from the pubis in monotremes and marsupials. They were initially thought to support the marsupium, i.e. the pouch in marsupials and echidnas, but this view is contended given their presence in species that do not have a pouch. They may indeed be related to the presence of an indented pouch 'region' if not a full pouch, but they have also been implicated in gait and locomotion. Specifically, the epipubic bones extend into the superficial layers of hypaxial abdominal musculature, bearing a series of attachments and connections to the body wall, the midline, and the femur, and contribute to a "cross-couplet" lever system during locomotion that is more similar to the reptilian condition than the unilateral muscle activation pattern of eutherian mammals (Vázquez - Molinero, Martin et al. 2001, Reilly and White 2003, Reilly, McElroy et al. 2009).

Despite their uncertain function, it is likely that epipubic bones preclude trans-cyclic gestation by compromising pliability of the abdominal wall, which is essential for accommodation of a growing fetus for an extended duration. Extant eutherians lack epipubic bones. Their loss was probably one of the factors that made trans-cyclic gestation possible or their loss was driven by the need to accommodate numerous large-sized fetuses. Fossils of stem eutherians, however, do possess the epipubic bones, as do stem metatherians and stem therians - unambiguously indicating their 
homology across all these clades (Novacek, Rougier et al. 1997, Vázquez - Molinero, Martin et al. 2001). This indicates at least that these elements persisted until very near to, and perhaps into, the extant eutherian radiation, adding support for the argument that ancestral eutherian likely gave birth to small neonates and have had intra-cyclic gestation.

Taken together, these data suggest that, despite its universality in Eutheria, transcyclic gestation evolved independently several times after the eutherian radiation that gave rise to the major clades of placental mammals.

\section{Integration of our model in the narrative of mammalian evolution}

Below, we insert the ideas postulated above into the account of mammalian evolution, as currently understood (see Figure 6).

The eutherian ancestor was a very small mammal and gave birth to altricial neonates after a short gestation period. Gestation was intra-cyclic, and contained within the luteal phase of the cycle, as it is in opossum and many other marsupials. The embryo remained unattached for most of gestation, and implantation took place only toward the end of the pregnancy. This is similar to the scenario in extant marsupials such as opossums, and it is likely to have been the case in the marsupial ancestor as well as the therian ancestor. The key difference to the situation in marsupials was that, in the eutherian ancestor, implantation was invasive. This suggests that invasive placentation, following the destruction of endometrial luminal epithelium, elicited an inflammatory response in the endometrium. Tissue damage is known to lead to the activation of tissue resident fibroblasts, which participate in the inflammatory reaction similarly to tissue macrophages (Smith, Smith et al. 1997) and leads to the activation of FOX01 protein (Alikhani, Alikhani et al. 2005), a transcription factor known to be critical for decidualization (Gellersen and Brosens 2014). We thus suggest that DSC originated from ESF by converting the pro-inflammatory activated fibroblasts of the endometrial stroma into a specialized stromal cell type that participates in the inflammatory implantation process but changes the nature of the response to an anti-inflammatory state that is compatible with accommodating the conceptus. Some of the mechanisms of the switch from pro- to anti-inflammatory state have been identified; for instance the bi-phasic expression of IL-33 (Interleukin 33) related signaling molecules in human endometrial stromal fibroblasts during decidualization (Salker, Nautiyal et al. 2012).

In our model the eutherian ancestor had intra-cyclic gestation, parturition ensued soon after a short phase of invasive placentation. In this scenario, the role of DSC was limited to the peri-implantation period, possibly to modulate the inflammatory stimulus emanating from the blastocyst to limit tissue damage to the uterus.

Extinction of large terrestrial dinosaurs (excepting the small-bodied ancestors of the major avian lineages) at the Creataceous-Paleogene boundary (K-Pg), 65 million 
years ago, may have opened up ecological niches for mammals, leading to a mammalian radiation and several independent trends of increase in body size. Ancestors of the four major clades of eutherian mammals already existed before this mass extinction event (dos Reis, Inoue et al. 2012, but see O'Leary, Bloch et al. 2013 for an alternate hypothesis); therefore eutherian body size increased independently in several lineages after the K-Pg boundary.

Consequently, we hypothesize, that long, trans-cyclic gestation also evolved in concert with and to accommodate the increase in body size, independently in major eutherian lineages. Independent evolution of trans-cyclic gestation in turn makes it likely that different functional strategies evolved to sustain gestation. Trans-cyclic gestation can be thought of as the intercalation of a new phase of pregnancy between implantation and parturition (see Figure 4) in the ancestral mode of pregnancy hypothesized above. Addition of this phase necessitated resolution of immunological and physiological challenges of a long pregnancy that is associated with an invasive placenta. According to our model these challenges arose independently in various eutherian lineages, and so did the mechanisms of their resolutions. The resolution, in Euarchontoglires alone, involved extended life span of DSC, together with the acquisition of new functions related to maintenance of the trans-cyclic gestation. It is not fully understood how these challenges were resolved in Xenarthra and Afrotheria in a manner that does not involve DSC. It is possible that in Laurasiatheria, at least, the immunological challenge was tackled in part by loss of invasive placentation (Carter and Enders 2013).

\section{Alternative models explaining reproductive variation}

There is tremendous variation in the morphology and physiology of the fetalmaternal interface in therian mammals. Placenta is the most variable organ, at least in Theria, despite the fact that it serves the same basic function in all lineages. A number of explanations have been put forth to explain this variation.

Parent-offspring conflict is often invoked as a driver of diversity in placental structures (Crespi and Semeniuk 2004). In this model, placental diversity is the result of a constant tug-of-war between the mother and the fetus, particularly over characters related to resource transfer and the timing of parturition. While this argument may apply to the diversity within narrower clades of eutherians, we argue that the broad pattern of diversity across eutherians does not need a selective explanation, because the trans-cyclic mode of pregnancy is likely not homologous across major eutherian clades. Convergent origin of trans-cyclical gestation by different mechanisms is sufficient to explain diversity at this level of comparison.

Another possible explanation of mechanistic diversification is developmental systems drift i.e. variation in the developmental mechanisms of homologous characters (Weiss and Fullerton 2000, True and Haag 2001). One model for developmental system drift is the Selection-Pleiotropy-Compensation (SPC) model (Johnson and Porter 2001, Pavlicev and Wagner 2012). The SPC model assumes that 
selection of an adaptive mutation brings with it negative pleiotropic consequences, which are subsequently resolved by compensatory mutations. SPC model has been proposed as an explanation for many puzzling phenomena including developmental systems drift. According to SPC model, development of homologous characters, when evolving under a selection for compensating mutations, can turn out to be surprisingly variable among species. It can be argued that eutherian pregnancy is as variable as it is because it has been undergoing developmental systems drift under the SPC mode of evolution. The model proposed here provides an alternative explanation. The SPC model aims at explaining why homologous traits can be produced by different mechanisms. Again, the alternative is that the trait, transcyclical gestation, is not homologous across the major clades of eutherian mammals and thus it is not surprising that it is produced by different mechanisms.

We suspect that all three of these explanations; parent-offspring conflict, developmental systems drift and independent derivation; are at work in producing the variation among modes of eutherian gestation, but at this point it is not clear at what taxonomic levels these different mechanisms act. Parent-offspring conflict and SPC perhaps explain the variation in the shared derived phase of gestation i.e. the ancestral short post-implantation phase of gestation (see figure 4) as well as variation found among more closely related animals. Variation in the later stages of pregnancy that were independently acquired in various lineages can be explained by independent origination. Swaggart and colleagues (Swaggart, Pavlicev et al. 2015) point out that during embryonic development, organogenesis is generally completed by the time of luteolysis. For example, in mouse, CL persist till the end of gestation and the fetuses are altricial at the time of birth, while in human luteolysis takes place around 8-12 weeks of gestation coinciding with the completion of organogenesis (i.e. the fetus is roughly in the stage of development that mouse fetuses are at the time of birth) which is followed by a long phase of intrauterine growth and maturation. Swaggart and colleagues suggest that mouse gestation is homologous to only the first trimester of human gestation. The variation between mouse (and potentially other altricial rodent species) gestation and the first trimester of human gestation is probably better explained by SPC and parentoffspring conflict than by our model, while the variation between later stages of gestation of precocial mammals like guinea pig, and second and third trimesters of human gestation are probably better explained by the assumption of independent origins. The evaluation of these ideas requires more detailed analysis of gestational variation than is the scope of this paper.

\section{Conclusion}

In light of the their critical immune and endocrine functions to support pregnancy in rodents and primates, it is surprising that DSC in xenarthrans, afrotherians and some laurasiatherians, while present during implantation, are lost in later stages of pregnancy. Here we explained this pattern by proposing that DSC in the eutherian ancestor played a role only in the peri-implantation period, perhaps to modify the inflammatory reaction elicited by the process of invasive implantation. Their role in 
later gestation is not shared among the major eutherian lineages, and probably was acquired in the stem lineage of Euarchontoglires.

An obvious question arising from the model proposed here is how the fetalmaternal immune tolerance is mediated in Xenarthra and Afrotheria as for the majority of their gestation proceeds without any aid from DSC. Our understanding of this phenomenon is limited at the moment. One model, put forward by Enders and Welsh (Enders and Welsh 1993), suggests that one of the strategies is to limit the contact between the endometrial stroma and the trophoblast. More work is certainly required on this front.

Finally, an implication of our model is that research on the evolutionary origin of DSC will benefit most from understanding the biology of implantation and the role of DSC in that phase of gestation rather than their role in the maintenance of transcyclic gestation. 


\section{Figure captions}

Figure 1. Phylogeny of mammals. (a) Phylogenetic relationship between extant mammal species. Four major clades of Eutheria are coloured differently. Geological time scale is presented on the right hand side. Names of the species discussed in detail in this article are in bold typeface. The tree was drawn based on branchlengths from (dos Reis, Inoue et al. 2012). The relationship between Xenarthra, Afrotherina and Boreoeutheria is not clearly resolved so far. Three plausible relationships are shown in (b)(c) and (d).

Figure 2. Evolution of mode of placentation according to Wildman and colleagues (Wildman, Chen et al. 2006). The tree presents the results of phylogenetic ancestral state reconstruction of mode of placentation. The tree topology used for this analysis by the authors is that in Figure 1(c). Note that the reconstructed mode for the eutherian ancestral node is hemochorial.

Figure 3. Decidual stromal cells decrease over the length of gestation. (a) Rat: thickness of decidual layer is plotted against day of gestation. Thickness of all three layers of decidua decreases over time. Data for anti-mesometrial and lateral decidual thickness was obtained from (Dickson 1979) and data for mesometrial decidual thickness was obtained from (Furukawa, Hayashi et al. 2011). (b)(c) Armadillo: histological preparations of armadillo fetal-maternal interface stained for cytokeratin (brown). Cytokeratin marks trophoblast cells and glandular and luminal epithelia of the endometrium. In the early stage, the peri-implantation phase, endometrial stroma is present, and the stromal cells have DSC morphology. In the later stage, note the absence of endometrial layer between the placental villi and myometrium. (d)(e) Hyrax: Haematoxylin and Eosin stained slides of fetal-maternal interface of hyrax from Mossman Collection. Thickness of decidual layer in the early stage uterus (uterine diameter $8 \mathrm{~mm}$ ) is $1850 \mu$, which reduces to $750 \mu$ in a later stage uterus (uterine diameter $13 \mathrm{~mm})$. (f)(g) Tenrec: fetal-maternal interface from an early stage of gestation (crown-rump length 5-8mm), before the formation of definitive placenta, with permission, from (Carter, Blankenship et al. 2005) and a later stage of gestation (crown-rump length $58 \mathrm{~mm}$ ), after the formation of definitive placentation, with permission, from (Carter, Blankenship et al. 2004). Note that the endometrial stroma is present in the early stage, but completely disappears in the later stage. These images have been reproduced with permission from the publisher, and cropped to show the relevant parts of the images. Myo $=$ myometrium, $\mathrm{Tr}=$ Trophoblast, Endo.S. = Endometrial stroma Scale-bars on (b), (c) and (f) are $100 \mu$, and (g) is magnified 12x.

Figure 4. Intra-cyclic and trans-cyclic gestation. Intra-cyclic gestation is shorter than the sterile sexual cycle. Trans-cyclic gestation is longer than the sterile sexual cycle. Trans-cyclic gestation can be interpreted to have a new phase of gestation intercalated between implantation and parturition. 
Figure 5. Divergent mechanisms maintain trans-cyclic gestation in eutherian mammals. Mechanisms of maintenance of trans-cyclic gestation (sustained progesterone production beyond the length of sterile sexual cycle) are grouped into four categories: luteotropic signals, anti-luteolytic signals, shift in the organ of progesterone production, and other mechanisms. Note the remarkable diversity of the mechanisms. Roughly, Euarchontoglires use luteotropic signals and artiodactyls use anti-luteolytic signals.

$\mathrm{CL}=$ corpus luteum, $\mathrm{aCL}=$ accessory corpora lutea, $\mathrm{PRL}=$ prolactin, $\mathrm{hCG}=$ human chorionic gonadotropin, eCG = equine chorionic gonadotropin, E2 = estradiol, IFN- $\tau$ $=$ interferon-tau, $\mathrm{P} 4=$ progesterone.

Figure 6. Model for the evolution of eutherian gestation. Horizontal bars on the branches of the tree indicate evolutionary changes. Note the multiple origins of trans-cyclic gestation in Eutheria. The grey box indicates whether implantation and maintenance of trans-cyclic gestation are dependent on or independent of DSC. Intra-clade variation clearly exists, but here we have tried to show the most prominent patterns.

\section{Acknowledgements}

We would like to thank the Zoological Museum at University of Wisconsin-Madison for lending to us histological preparations from the Mossman Developmental Collection. Research into the evolution of the decidual cell type is funded by the John Templeton Foundation grant number 54860 "How new cell types arise in evolution". Other research in the lab is funded by NSF grant IRES\# 14-001273, as well as the Yale University Science Development Fund. 


\section{References}

Abrahamsohn, P. A. and T. M. Zorn (1993). "Implantation and decidualization in rodents." 」 Exp Zool 266(6): 603-628.

Alikhani, M., Z. Alikhani and D. T. Graves (2005). "FOXO1 functions as a master switch that regulates gene expression necessary for tumor necrosis factor-induced fibroblast apoptosis." J Biol Chem 280(13): 12096-12102.

Allen, W. (1984). "Hormonal control of early pregnancy in the mare." Animal Reproduction Science 7(1): 283-304.

Arendt, D. (2008). "The evolution of cell types in animals: emerging principles from molecular studies." Nat Rev Genet 9(11): 868-882.

Bazer, F. W. and T. E. Spencer (2011). Chapter 5 - Hormones and Pregnancy in Eutherian Mammals. Hormones and Reproduction of Vertebrates. D. O. N. H. Lopez. London, Academic Press: 73-94.

Becher, H. (1931). "Placenta und Uterusschleimhaut von Tamandua tetradactyla (Myrmecophaga)." Gegenbaurs Morphologisches Jahrbuch 67: 381 - 458.

Benirschke, K. (2011). "Comparative Placentation." Retrieved December 13, 2015, from http://placentation.ucsd.edu/homefs.html.

Brar, A. K., S. Handwerger, C. A. Kessler and B. J. Aronow (2001). "Gene induction and categorical reprogramming during in vitro human endometrial fibroblast decidualization." Physiol Genomics 7(2): 135-148.

Brayer, K. J., V. J. Lynch and G. P. Wagner (2011). "Evolution of a derived proteinprotein interaction between HoxA11 and Foxo1a in mammals caused by changes in intramolecular regulation." Proceedings of the National Academy of Sciences of the United States of America 108(32): E414-E420.

Carter, A. M., T. N. Blankenship, H. Kunzle and A. C. Enders (2004). "Structure of the definitive placenta of the tenrec, Echinops telfairi." Placenta 25(2-3): 218-232.

Carter, A. M., T. N. Blankenship, H. Kunzle and A. C. Enders (2005). "Development of the haemophagous region and labyrinth of the placenta of the tenrec, Echinops telfairi." Placenta 26(2-3): 251-261.

Carter, A. M. and A. C. Enders (2013). "The Evolution of Epitheliochorial Placentation." Annual Review of Animal Biosciences 1(1): 443-467.

Christian, M., X. Zhang, T. Schneider-Merck, T. G. Unterman, B. Gellersen, J. O. White and J. J. Brosens (2002). "Cyclic AMP-induced forkhead transcription factor, FKHR, 
cooperates with CCAAT/enhancer-binding protein beta in differentiating human endometrial stromal cells." 」Biol Chem 277(23): 20825-20832.

Clauss, M., M. T. Dittmann, D. W. H. Muller, P. Zerbe and D. Codron (2014). "Low scaling of a life history variable: Analysing eutherian gestation periods with and without phylogeny-informed statistics." Mammalian Biology 79(1): 9-16.

Collins, M. K., C. S. Tay and A. Erlebacher (2009). "Dendritic cell entrapment within the pregnant uterus inhibits immune surveillance of the maternal/fetal interface in mice." 」 Clin Invest 119(7): 2062-2073.

Cooper, L. N., J. Thewissen, S. Bajpai and B. Tiwari (2012). "Postcranial morphology and locomotion of the Eocene raoellid Indohyus (Artiodactyla: Mammalia)." Historical Biology 24(3): 279-310.

Crespi, B. and C. Semeniuk (2004). "Parent-offspring conflict in the evolution of vertebrate reproductive mode." American Naturalist 163(5): 635-653.

Dai, D., B. C. Moulton and T. F. Ogle (2000). "Regression of the decidualized mesometrium and decidual cell apoptosis are associated with a shift in expression of Bcl2 family members." Biol Reprod 63(1): 188-195.

Dekel, N., Y. Gnainsky, I. Granot, K. Racicot and G. Mor (2014). "The role of inflammation for a successful implantation." Am J Reprod Immunol 72(2): 141-147.

Dickson, A. D. (1979). "The disappearance of the decidua capsularis and Reichert's membrane in the mouse." LAnat 129(Pt 3): 571-577.

dos Reis, M., J. Inoue, M. Hasegawa, R. J. Asher, P. C. Donoghue and Z. Yang (2012). "Phylogenomic datasets provide both precision and accuracy in estimating the timescale of placental mammal phylogeny." Proc Biol Sci 279(1742): 3491-3500.

Elliot, M. G. and B. J. Crespi (2009). "Phylogenetic evidence for early hemochorial placentation in eutheria." Placenta 30(11): 949-967.

Enders, A. C., G. D. Buchanan and R. V. Talmage (1958). "Histological and Histochemical Observations on the Armadillo Uterus during the Delayed and Postimplantation Periods." Anatomical Record 130(4): 639-657.

Enders, A. C. and A. M. Carter (2012). "Review: The evolving placenta: Different developmental paths to a hemochorial relationship." Placenta 33, Supplement: S92-S98.

Enders, A. C. and A. O. Welsh (1993). "Structural Interactions of Trophoblast and Uterus during Hemochorial Placenta Formation." Journal of Experimental Zoology 266(6): 578-587. 
Erlebacher, A. (2013). "Immunology of the Maternal-Fetal Interface." Annual Review of Immunology, Vol 31 31: 387-411.

Freyer, C., U. Zeller and M. B. Renfree (2003). "The marsupial placenta: A phylogenetic analysis." Journal of Experimental Zoology Part a-Comparative Experimental Biology 299A(1): 59-77.

Furukawa, S., S. Hayashi, K. Usuda, M. Abe, S. Hagio and I. Ogawa (2011).

"Toxicological pathology in the rat placenta." L Toxicol Pathol 24(2): 95-111.

Gellersen, B. and J. J. Brosens (2014). "Cyclic decidualization of the human endometrium in reproductive health and failure." Endocr Rev 35(6): 851-905.

Gersell, D. and F. Kraus (2011). Diseases of the Placenta. Blaustein's Pathology of the Female Genital Tract. R. Kurman, L. Ellenson and B. Ronnett, Springer US: 999-1073.

Gopalakrishna, A. and M. S. Khaparde (1978). "Development of the foetal membranes and placentation in the Indian false vampire bat,Megaderma lyra lyra (Geoffroy)." Proceedings of the Indian Academy of Sciences - Section B, Animal Sciences 87(9): 179-194.

Grosser, 0. (1927). Fruhentwicklung, Eihautbildung und Placentatsion des Menschen und der Saugetiere. Munchen, J. F. Bermann.

Gu, Y., G. M. Jow, B. C. Moulton, C. Lee, J. A. Sensibar, O. K. Park-Sarge, T. J. Chen and G. Gibori (1994). "Apoptosis in decidual tissue regression and reorganization." Endocrinology 135(3): 1272-1279.

Hone, D. W. and M. J. Benton (2005). "The evolution of large size: how does Cope's Rule work?" Trends in Ecology \& Evolution 20(1): 4-6.

Johnson, N. A. and A. H. Porter (2001). "Towards a new synthesis: population genetics and evolutionary developmental biology." Genetica 112-113: 45 - 58.

Keskin, D. B., D. S. J. Allan, B. Rybalov, M. M. Andzelm, J. N. H. Stern, H. D. Kopcow, L. A. Koopman and J. L. Strominger (2007). "TGF beta promotes conversion of CD16(+) peripheral blood NK cells into CD16(-) NK cells with similarities to decidual NK cells." Proceedings of the National Academy of Sciences of the United States of America 104(9): 3378-3383.

Kielan-Jaworowska, Z., R. L. Cifelli and Z.-X. Luo (2004). Mammals From the Age of Dinosaurs: Origins, Evolution, and Structure. New York, Columbia University Press.

Kihlstrom, J. E. (1972). "Period of Gestation and Body-Weight in Some Placental Mammals." Comparative Biochemistry and Physiology 43(Na3): 673-\&. 
Kin, K., J. Maziarz and G. P. Wagner (2014). "Immunohistological study of the endometrial stromal fibroblasts in the opossum, Monodelphis domestica: evidence for homology with eutherian stromal fibroblasts." Biol Reprod 90(5): 111.

Kitaya, K., J. Yasuda, I. Yagi, Y. Tada, S. Fushiki and H. Honjo (2000). "IL-15 expression at human endometrium and decidua." Biology of Reproduction 63(3): 683-687.

Labhsetwar, A. P. and A. C. Enders (1968). "Progesterone in Corpus Luteum and Placenta of Armadillo Dasypus Novemcinctus." Journal of Reproduction and Fertility 16(3): 381-\&.

Lueders, I., C. Niemuller, P. Rich, C. Gray, R. Hermes, F. Goeritz and T. B. Hildebrandt (2012). "Gestating for 22 months: luteal development and pregnancy maintenance in elephants." Proceedings of the Royal Society B-Biological Sciences 279(1743): 3687-3696.

Lynch, V. J., K. Brayer, B. Gellersen and G. P. Wagner (2009). "HoxA-11 and F0X01A cooperate to regulate decidual prolactin expression: towards inferring the core transcriptional regulators of decidual genes." PLoS One 4(9): e6845.

Lynch, V. J., G. May and G. P. Wagner (2011). "Regulatory evolution through divergence of a phosphoswitch in the transcription factor CEBPB." Nature 480(7377): 383-386.

Macklon, N. S. and J. J. Brosens (2014). "The human endometrium as a sensor of embryo quality." Biol Reprod 91(4): 98.

Malassine, A. and R. Leiser (1984). "Morphogenesis and Fine-Structure of the near Term Placenta of Talpa Europaea .1. Endotheliochorial Labyrinth." Placenta 5(2): 145-158.

Martin, R. (2008). "Evolution of Placentation in Primates: Implications of Mammalian Phylogeny." Evolutionary Biology 35(2): 125-145.

Martin, R. D. and A. M. Maclarnon (1985). "Gestation Period, Neonatal Size and Maternal Investment in Placental Mammals." Nature 313(5999): 220-223.

McAllan, B. M. (2011). Chapter 10 - Reproductive Endocrinology of Prototherians and Metatherians. Hormones and Reproduction of Vertebrates. D. O. N. H. Lopez. London, Academic Press: 195-214.

Mess, A. and A. M. Carter (2006). "Evolutionary transformations of fetal membrane characters in Eutheria with special reference to Afrotheria." LExp Zool B Mol Dev Evol 306(2): 140-163. 
Mor, G., I. Cardenas, V. Abrahams and S. Guller (2011). "Inflammation and pregnancy: the role of the immune system at the implantation site." Annals of the New York Academy of Sciences 1221(1): 80-87.

Mossman, H. W. (1937). "Comparative morphogenesis of the fetal membranes and accessory uterine structures." Contributions to Embryology 26(158): 133-137.

Mossman, H. W. (1987). Vertebrate fetal membranes : comparative ontogeny and morphology, evolution, phylogenetic significance, basic functions, research opportunities. New Brunswick, N.J., Rutgers University Press.

Nakakura, K., N. M. Czekala, B. L. Lasley and K. Benirschke (1982). "Fetal Maternal Gradients of Steroid-Hormones in the 9-Banded Armadillo (DasypusNovemcinctus)." Journal of Reproduction and Fertility 66(2): 635-643.

Nancy, P., E. Tagliani, C. S. Tay, P. Asp, D. E. Levy and A. Erlebacher (2012). "Chemokine gene silencing in decidual stromal cells limits $\mathrm{T}$ cell access to the maternal-fetal interface." Science 336(6086): 1317-1321.

Novacek, M. J., G. W. Rougier, J. R. Wible, M. C. McKenna, D. Dashzeveg and I. Horovitz (1997). "Epipubic bones in eutherian mammals from the Late Cretaceous of Mongolia." Nature 389(6650): 483-486.

O'Leary, M. A., J. I. Bloch, J. J. Flynn, T. J. Gaudin, A. Giallombardo, N. P. Giannini, S. L. Goldberg, B. P. Kraatz, Z. X. Luo, J. Meng, X. Ni, M. J. Novacek, F. A. Perini, Z. S. Randall, G. W. Rougier, E. J. Sargis, M. T. Silcox, N. B. Simmons, M. Spaulding, P. M. Velazco, M. Weksler, J. R. Wible and A. L. Cirranello (2013). "The placental mammal ancestor and the post-K-Pg radiation of placentals." Science 339(6120): 662-667.

O'Leary, M. A., J. I. Bloch, J. J. Flynn, T. J. Gaudin, A. Giallombardo, N. P. Giannini, S. L. Goldberg, B. P. Kraatz, Z. X. Luo, J. Meng, X. J. Ni, M. J. Novacek, F. A. Perini, Z. S. Randall, G. W. Rougier, E. J. Sargis, M. T. Silcox, N. B. Simmons, M. Spaulding, P. M. Velazco, M. Weksler, J. R. Wible and A. L. Cirranello (2013). "The Placental Mammal Ancestor and the Post-K-Pg Radiation of Placentals." Science 339(6120): 662-667.

Pavlicev, M. and G. P. Wagner (2012). "A model of developmental evolution: selection, pleiotropy and compensation." Trends Ecol Evol 27(6): 316-322.

Portmann, A. (1938). Die Ontogenese der Säugetiere als Evolutionsproblem. Basel, S. Karger.

Reilly, S. M., E. J. McElroy and T. D. White (2009). "Abdominal muscle function in ventilation and locomotion in new world opossums and basal eutherians: Breathing and running with and without epipubic bones." \Morphol 270: 1014-1028.

Reilly, S. M. and T. D. White (2003). "Hypaxial motor patterns and the function of epipubic bones in primitive mammals." Science 299(5605): 400-402. 
Renfree, M. (1994). Endocrinology of Pregnancy, Parturition and Lactation in Marsupials. Marshall's Physiology of Reproduction. G. E. Lamming, Springer Netherlands: 677-766.

Rose, K. D. (1982). "Skeleton of Diacodexis, oldest known artiodactyl." Science 216(4546): 621-623.

Rose, K. D. and J. D. Archibald (2005). The rise of placental mammals: origins and relationships of the major extant clades, JHU Press.

Salker, M. S., J. Nautiyal, J. H. Steel, Z. Webster, S. Sucurovic, M. Nicou, Y. Singh, E. S. Lucas, K. Murakami, Y. W. Chan, S. James, Y. Abdallah, M. Christian, B. A. Croy, B. Mulac-Jericevic, S. Quenby and J. J. Brosens (2012). "Disordered IL-33/ST2 activation in decidualizing stromal cells prolongs uterine receptivity in women with recurrent pregnancy loss." PLoS One 7(12): e52252.

Shooner, C., P. L. Caron, G. Frechette-Frigon, V. Leblanc, M. C. Dery and E. Asselin (2005). "TGF-beta expression during rat pregnancy and activity on decidual cell survival." Reprod Biol Endocrinol 3: 20.

Simmons, N. B., K. L. Seymour, J. Habersetzer and G. F. Gunnell (2008). "Primitive Early Eocene bat from Wyoming and the evolution of flight and echolocation." Nature 451(7180): 818-821.

Smith, R. S., T. J. Smith, T. M. Blieden and R. P. Phipps (1997). "Fibroblasts as sentinel cells - Synthesis of chemokines and regulation of inflammation." American Journal of Pathology 151(2): 317-322.

Stansfield, F. J. and W. R. Allen (2012). "Luteal maintenance of pregnancy in the African elephant (Loxodonta africana)." Reproduction 143(6): 845-854.

Swaggart, K. A., M. Pavlicev and L. J. Muglia (2015). "Genomics of Preterm Birth." Cold Spring Harbor Perspectives in Medicine 5(2).

Tamura, I., Y. Ohkawa, T. Sato, M. Suyama, K. Jozaki, M. Okada, L. Lee, R. Maekawa, H. Asada, S. Sato, Y. Yamagata, H. Tamura and N. Sugino (2014). "Genome-wide analysis of histone modifications in human endometrial stromal cells." Mol Endocrinol 28(10): 1656-1669.

Thursby-Pelham, D. (1925). "The placentation of Hyrax capensis." Philosophical Transactions of the Royal Society of London Series B-Containing Papers of a Biological Character 213: 1-20.

True, J. R. and E. S. Haag (2001). "Developmental system drift and flexibility in evolutionary trajectories." Evolution \& Development 3(2): 109-119. 
Vacca, P., C. Vitale, E. Montaldo, R. Conte, C. Cantoni, E. Fulcheri, V. Darretta, L. Moretta and M. C. Mingari (2011). "CD34+ hematopoietic precursors are present in human decidua and differentiate into natural killer cells upon interaction with stromal cells." Proc Natl Acad Sci U S A 108(6): 2402-2407.

Vázquez - Molinero, R., T. Martin, M. Fischer and R. Frey (2001). "Comparative anatomical investigations of the postcranial skeleton of Henkelotherium guimarotae Krebs, 1991 (Eupantotheria, Mammalia) and their implications for its locomotion." Zoosystematics and Evolution 77(2): 207-216.

Wagner, G. P. and V. J. Lynch (2010). "Evolutionary novelties." Curr Biol 20(2): R4852.

Wang, H. and S. K. Dey (2006). "Roadmap to embryo implantation: clues from mouse models." Nat Rev Genet 7(3): 185-199.

Weiss, K. M. and S. M. Fullerton (2000). "Phenogenetic Drift and the Evolution of Genotype-Phenotype Relationships." Theoretical Population Biology 57(3): 187195.

Wildman, D. E., C. Y. Chen, O. Erez, L. I. Grossman, M. Goodman and R. Romero (2006). "Evolution of the mammalian placenta revealed by phylogenetic analysis." Proceedings of the National Academy of Sciences of the United States of America 103(9): 3203-3208.

Wimsatt, W. A. and A. C. Enders (1980). "Structure and Morphogenesis of the Uterus, Placenta, and Paraplacental Organs of the Neotropical Disc-Winged Bat ThyropteraTricolor-Spix (Microchiroptera, Thyropteridae)." American Journal of Anatomy 159(2): 209-243.

Wooding, F. B. P. and G. Burton (2008). Comparative placentation : structures, functions and evolution. Berlin, Springer. 
Figure 1

(a)

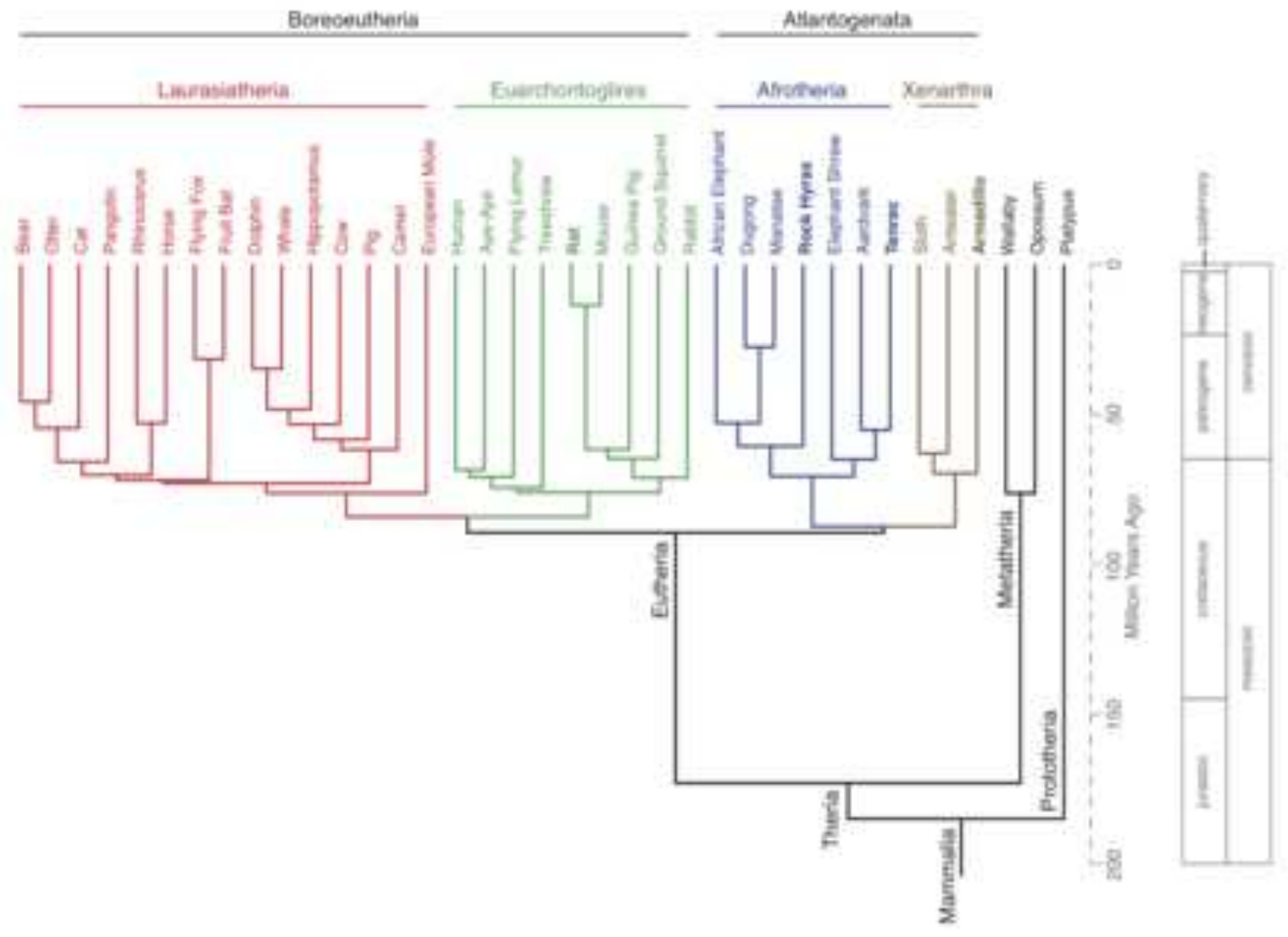

(b)

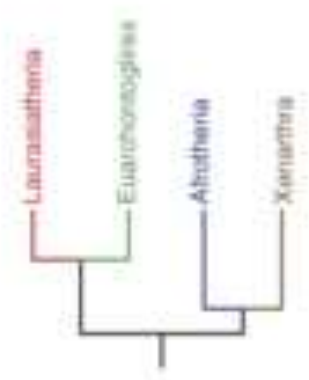

(c)

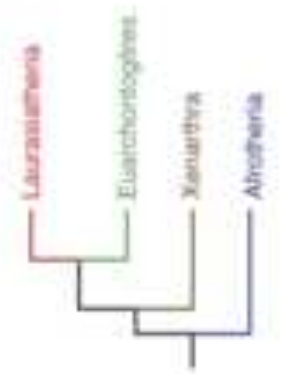

(d)

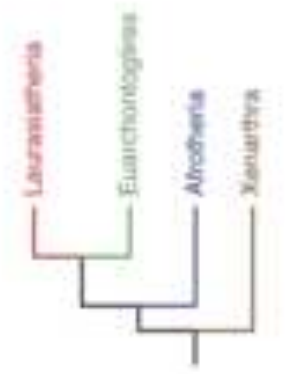




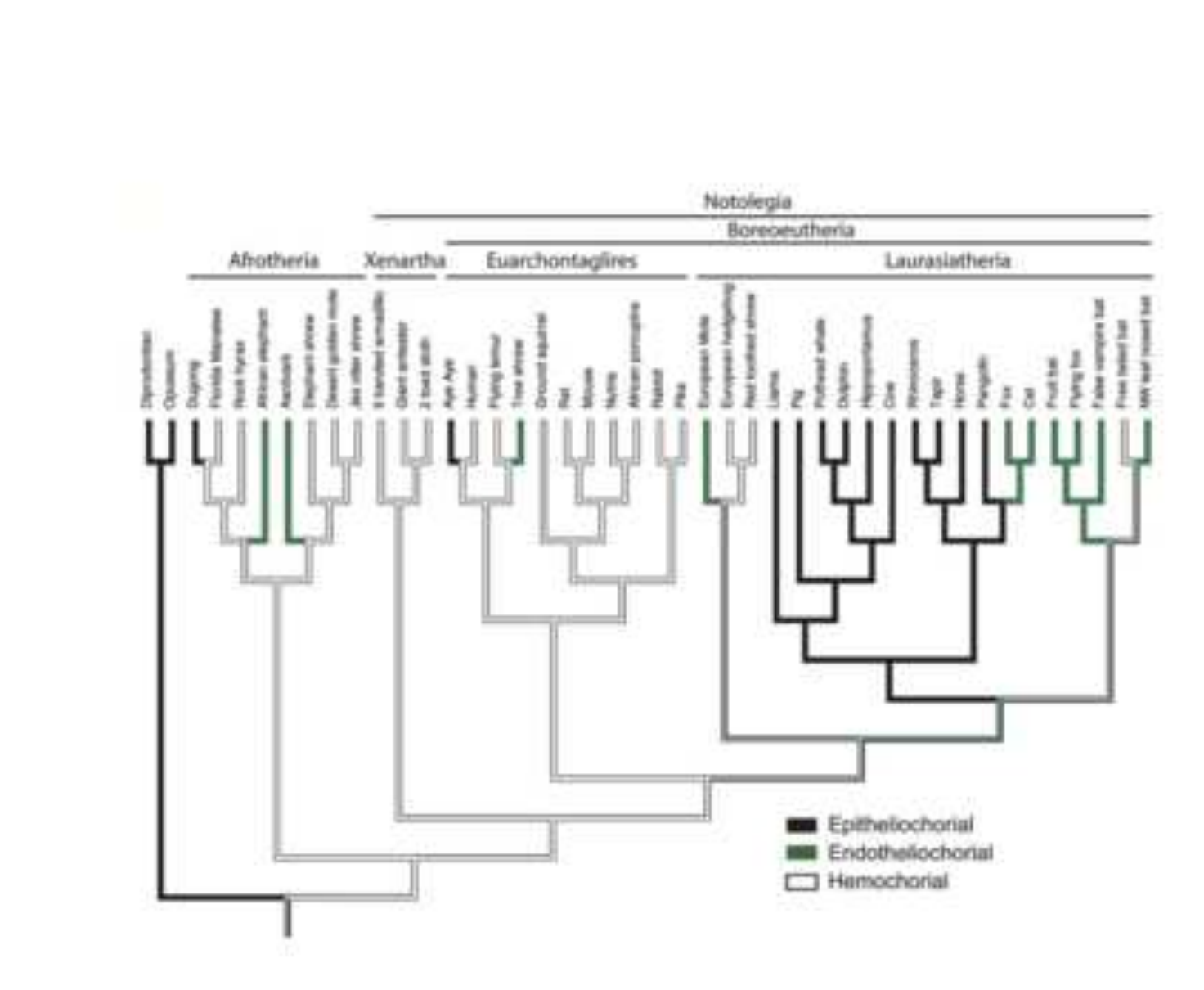

Figure 2

\section{Figure}

2
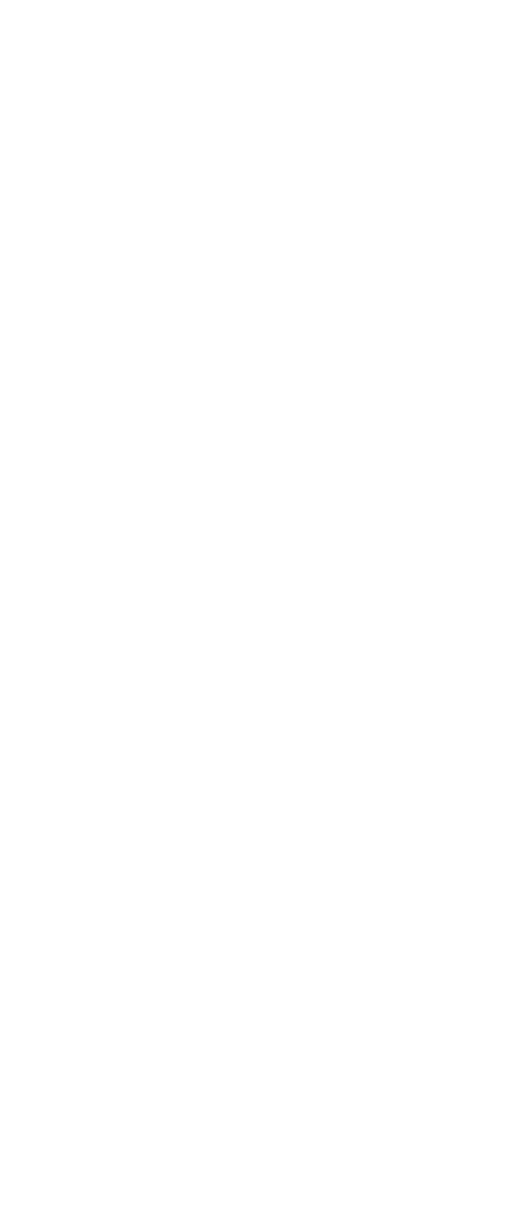
(a) Decidual thickness over time
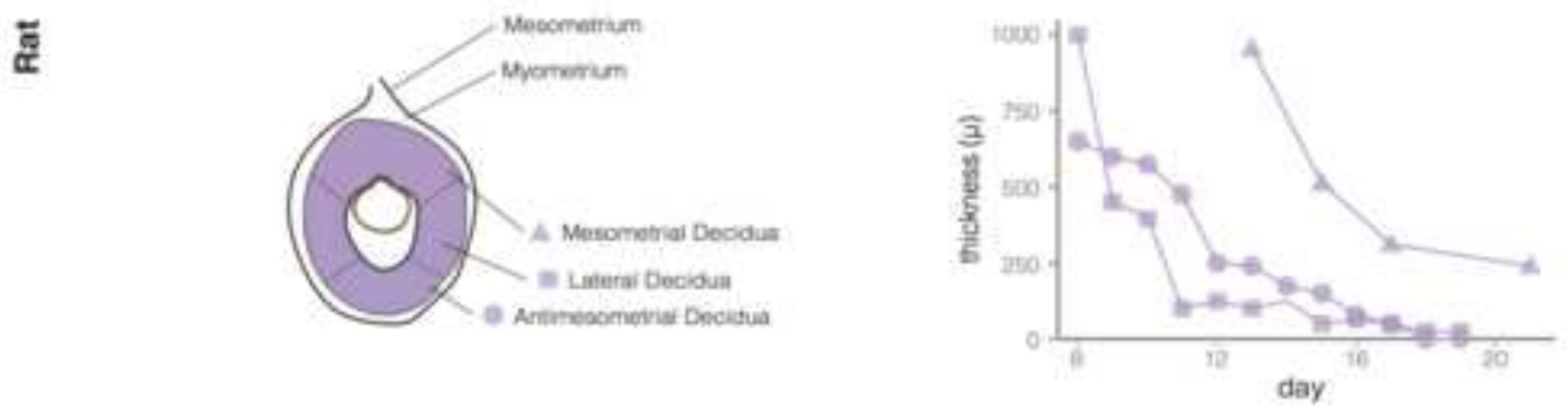

(b) Early

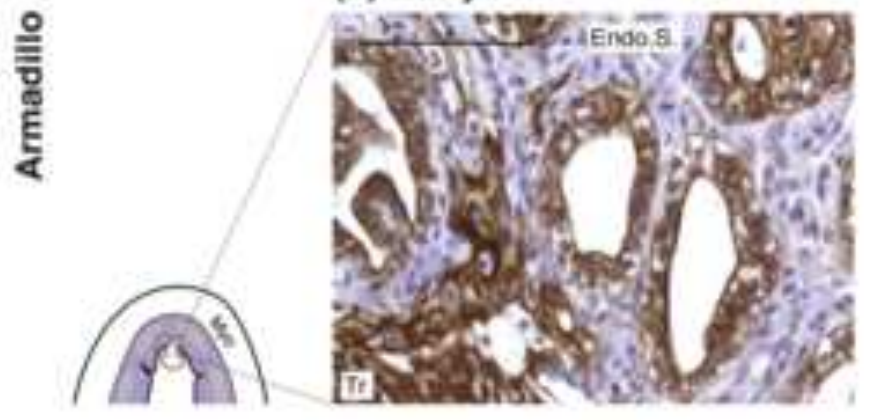

(c) Late
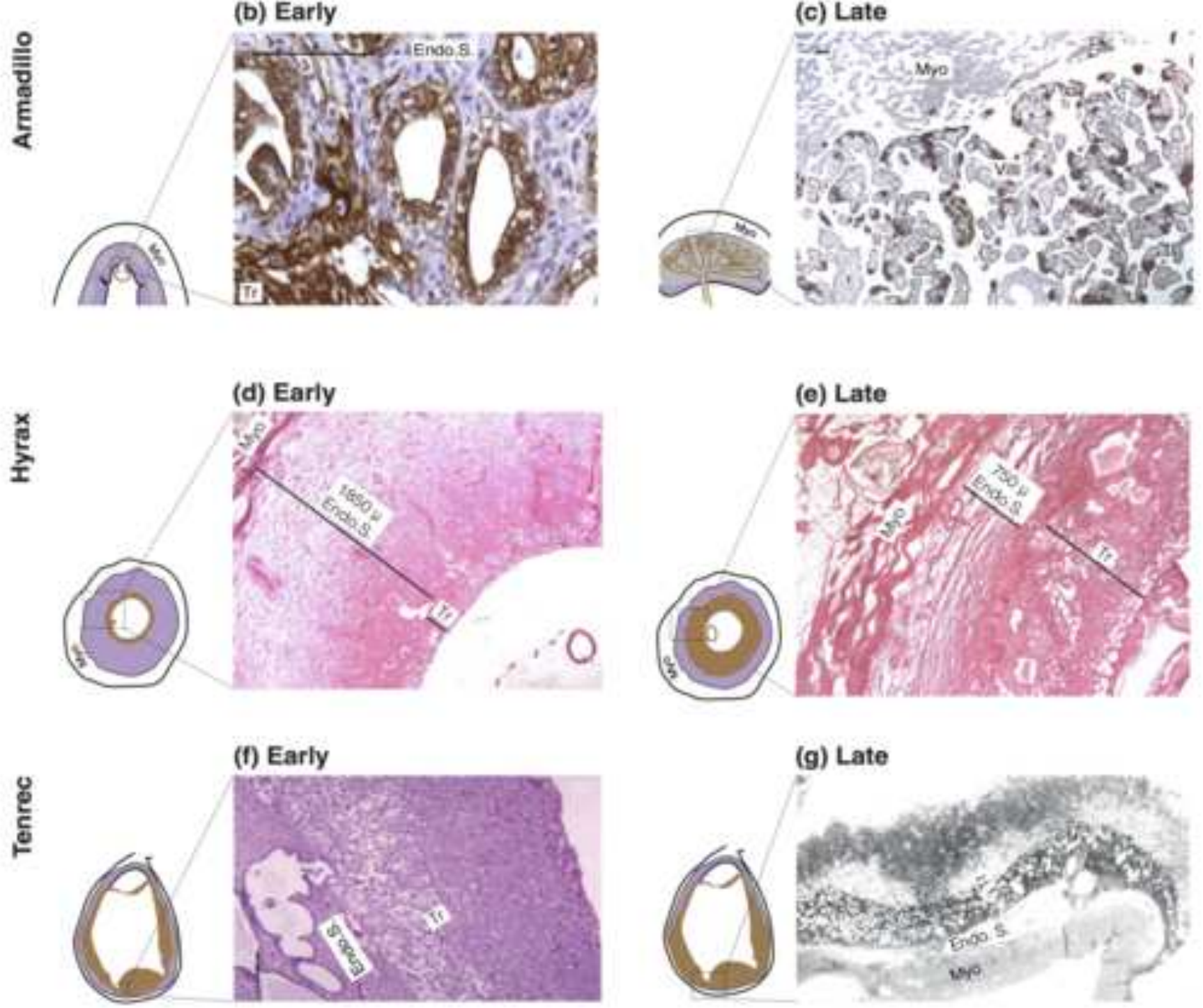

Erochoblast al Manemal Blood Sinus Endomatrial Stroma 


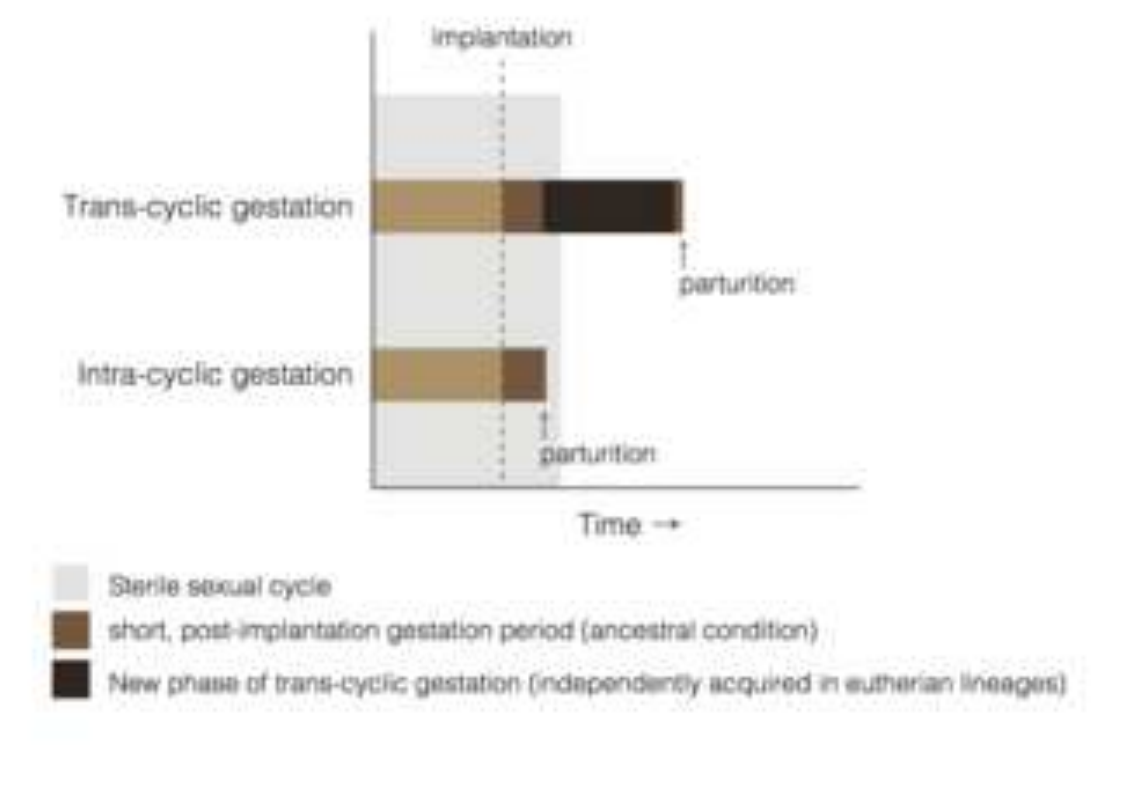

Sarile sexual cycle

short, post implantation gastation period (ancestral condition)

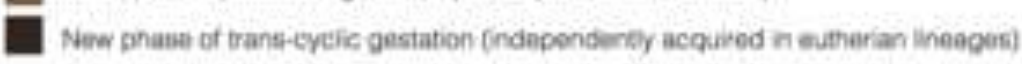

Figure 4

Time $\rightarrow$

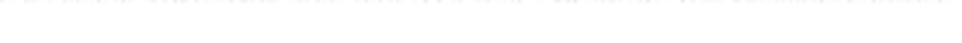

Figure 4

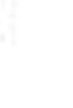
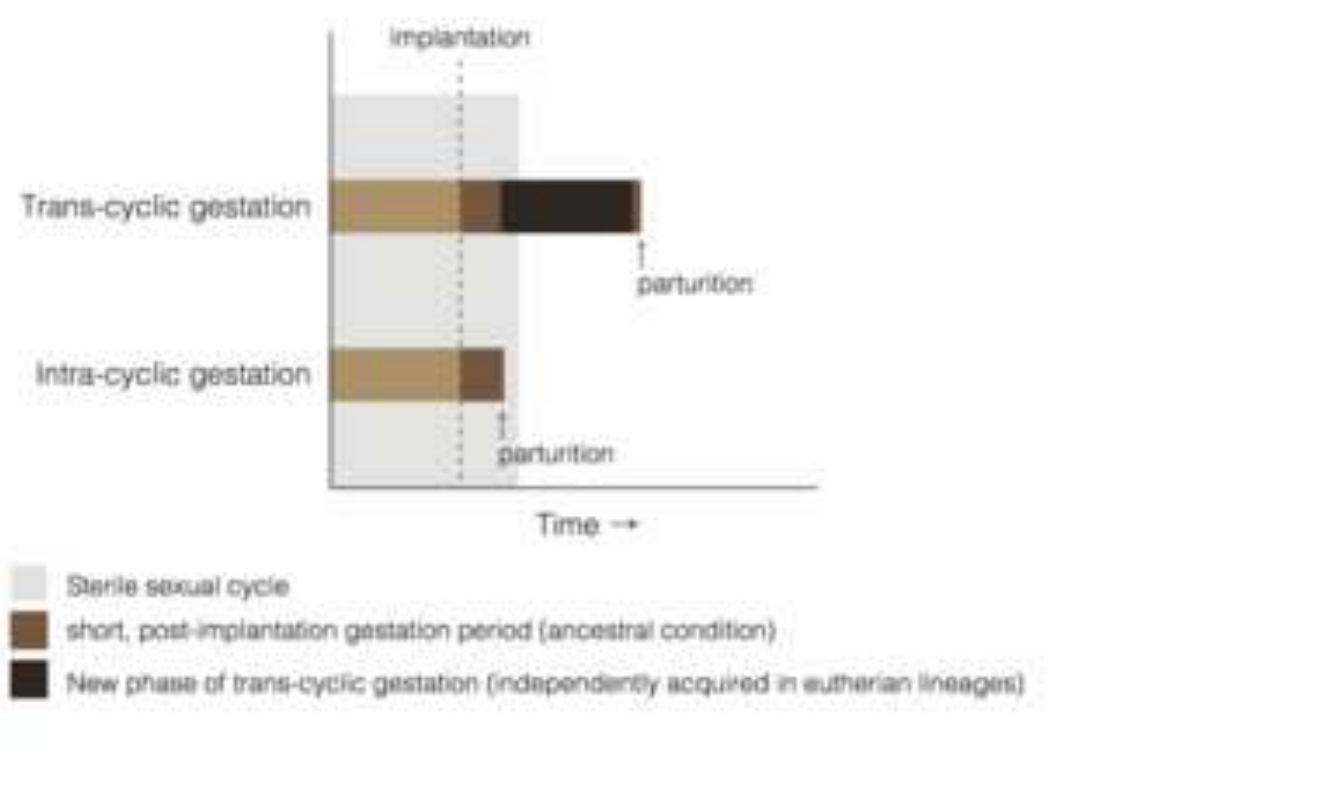


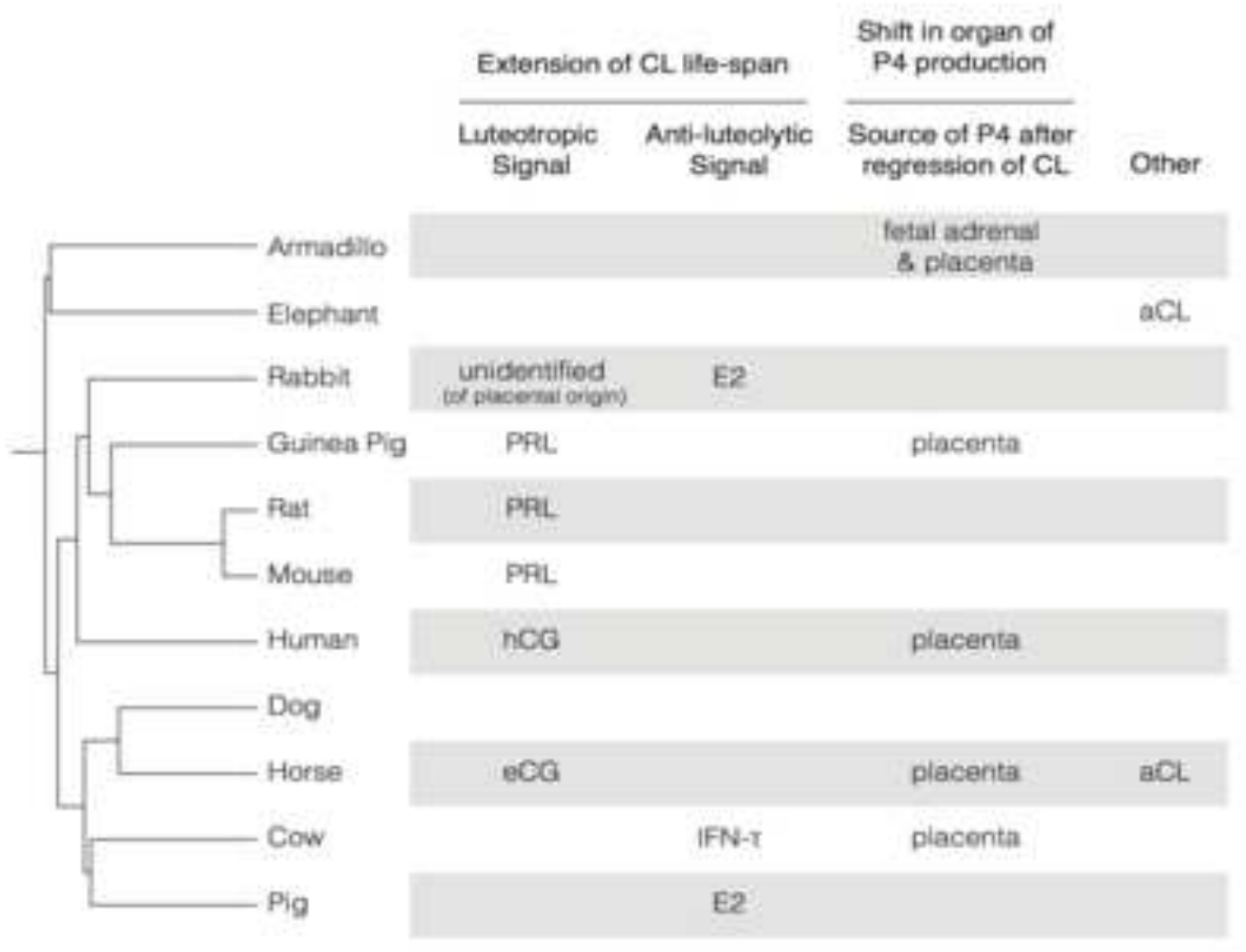

Figure 5

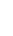
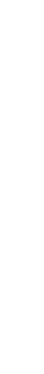

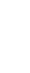

letal adrenal placente

\begin{tabular}{|c|c|c|c|}
\hline \multicolumn{2}{|c|}{ Extension of CL lite-span } & \multirow{2}{*}{$\begin{array}{l}\begin{array}{l}\text { Shitt in organ of } \\
\mathrm{P} 4 \text { production }\end{array} \\
\begin{array}{l}\text { Source of } \mathrm{P} 4 \text { affor } \\
\text { regression of } \mathrm{CL}\end{array}\end{array}$} & \multirow[b]{2}{*}{ Other } \\
\hline $\begin{array}{l}\text { Luteotropic } \\
\text { Signai }\end{array}$ & $\begin{array}{l}\text { Anti-luteolytic } \\
\text { Signal }\end{array}$ & & \\
\hline & \multicolumn{3}{|c|}{$\begin{array}{l}\text { fetal adrenal } \\
\text { \& placente }\end{array}$} \\
\hline & & & aCL. \\
\hline $\begin{array}{l}\text { unidentifiod } \\
\text { tot placenal orianis }\end{array}$ & $E 2:$ & & \\
\hline PRL & & piacenta & \\
\hline \multicolumn{4}{|l|}{ PPL } \\
\hline \multicolumn{4}{|l|}{ PAL } \\
\hline$n C G$ & & placenta & \\
\hline \multirow[t]{3}{*}{ eCG } & & placenta & $\mathrm{aCl}$ \\
\hline & IFN-T & placentn & \\
\hline & E2 & & \\
\hline
\end{tabular}

Shitt in organ of

Source of $\mathrm{P} 4$ affer

teotropic Signal regression of $\mathrm{CL}$.

Other 
